\title{
Visualising spatio-temporal modelling results: Getting modelling results into the hands of decision makers
}

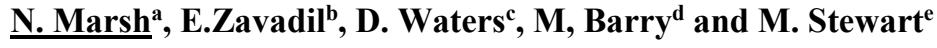 \\ ${ }^{a}$ Truii.com \\ ${ }^{b}$ Alluvium consulting \\ ${ }^{c}$ Queensland Department of Natural Resources and Mines: Principal scientist \\ ${ }^{d}$ BMTWBM: Technical and Innovation Manager \\ e Seqwater; Catchment Performance and planning Officer \\ Email: nick.marsh@truii.com
}

\begin{abstract}
Water quality models are routinely developed to support the natural resource management planning process. Each scenario of a water quality model generates a large volume of data. However the collective spatial and temporal elegance of the model output is usually summarised into simple coarse measures to allow straightforward document-based reports to communicate the key points to decision makers. This need to grossly simplify model results removes opportunities for decision makers and others involved in natural resource management to gain a greater appreciation of the underlying processes and interactions represented by the model.

By adopting a web based presentation of modelling results (compared to current document-based reporting), a 'progressive disclosure' approach can be adopted whereby high-level summaries can be linked to underlying results. This progressive disclosure approach allows decision makers and others to initially see the highest level summaries but then to also explore the underlying temporal and spatial richness of modelling results. This approach also allows a single reporting approach to suit an audience from a wide range of backgrounds. For example, senior managers may only wish to see a single summary table, but modellers and operational staff may need to access animated spatial representations of sediment and nutrient generation by sub catchment for particular climatic periods.

In this paper we describe a workflow for integrating models in the natural resource management process. We step through four case studies (one from the Great Barrier Reef catchments, two from South East Queensland and one from Victoria) where web based data visualisations have been used to deliver complex modelling results. These examples demonstrate the progressive disclosure approach for delivering sophisticated scientific understanding to different audiences. The case studies use data from a range of sources (observational data, SOURCE model output, TUFLOW model output, a spatially applied simulated annealing optimisation model and expert panel derived conceptual models).

The vast majority of the results generated through the modelling process never see the light of day, never get to a decision maker and ultimately never get to influence the process for which they were commissioned. Getting model results into the hands of decision makers adds value to the models and supports the education of the end users of model results
\end{abstract}

Keywords: Catchment modelling, data visualisation, web delivery, natural resource management 
Marsh et al., Visualising spatio-temporal modelling results: Getting modelling results into the hands of decision makers

\section{INTRODUCTION}

The natural resource management industry is data driven. The industry has a high reliance and well developed history of using measured and modelled data to support the planning and operationalization of natural resource management decisions (Cillers et al 2013). The environmental modelling community has created a large range of modelling tools to support natural resource management (Brugnach and Pahl-Wostl 2008). These tools have grown in sophistication. Catchment models that routinely handle many interacting processes are able to be provide useful and realistic scenario results. However, the methods of communicating model results to those that make decisions has changed very little.

To illustrate the value of data driven decision making from a business perspective, a survey of 330 public companies from North American showed a distribution of data driven management approaches. Those companies in the top third (i.e. the most data driven) were $5 \%$ more productive and $6 \%$ more profitable than their competitors (accounting for other factors) (McAfee and Brynjolfsson 2012). Using data to make decisions over and above raw skill and experience produces a measurably better outcome in the commercial sector.

It is not simply the need to be data driven, but how data is presented that can have a significant impact. Intuitively, a great chart trumps a table of numbers at communicating a key idea or trend. Pandey et al (2014) tested this hypothesis by running three alternative experiments across 720 individuals to test the persuasiveness of charts versus tables. The results show that for people who were not strongly polarised in their views (tables nor charts persuade zealots), charts were $8 \%$ more effective at converting views than the same data presented as a table. The key message here is that to be persuasive in delivering a data driven message, the results should be visualised and in a form that is easily consumed by the audience.

\subsection{Using models in Natural Resource Management Planning}

The typical process of integrating model results into the planning process is to produce a document-based report which simplifies the model results into key headline figures. While this approach gets the key points into the hands of decision makers, it does little to educate the decision makers on the underlying processes. It also misses an opportunity for the emergent properties of models to be realised. Those emergent properties are the broader, unspecified applications of the model which cannot be determined a priori. Decision makers, who are dealing with a large collection of competing priorities, are best placed to recognise these opportunities. However, they are not given the opportunity to do so because they are unable to explore the model scenarios within the context of their business environment.

We argue that it is time to present model results in a format that allows all users of the model results including decision makers, policy officers, scientists and other modellers to explore the data in a more natural learning and inquiring manner. Recent advances in cloud data storage, data services and web based data visualisation techniques present opportunities to deliver model results in a more intuitive forms that allow progressive disclosure and user exploration of data. Progressive disclosure is a software development approach whereby interfaces are designed to maintain the users focus by removing clutter, confusion and cognitive workload (Lidwell et al 2003). Natural learning is an educational term referring to an unstructured 'learning by doing' approach. Within the context of modelling to support natural resource management decision makers should be able to interactively explore data to better understand a model and how it can be applied to their business needs (natural learning). To facilitate the natural learning process, data delivery should adopt a progressive disclosure approach.

To explore the opportunities for a progressive disclosure approach to data presentation we first need to consider the different phases of the workflow where modelling supports natural resource management, and the relevant audience for each phase. When applying a modelling approach to natural resource management there are four basic phases of the workflow (Figure 1). The flow is from conceptualisation of the problem through to operationalisation of a solution. Each phase of the workflow engages a different audience and has a different measure of success (Table 1).

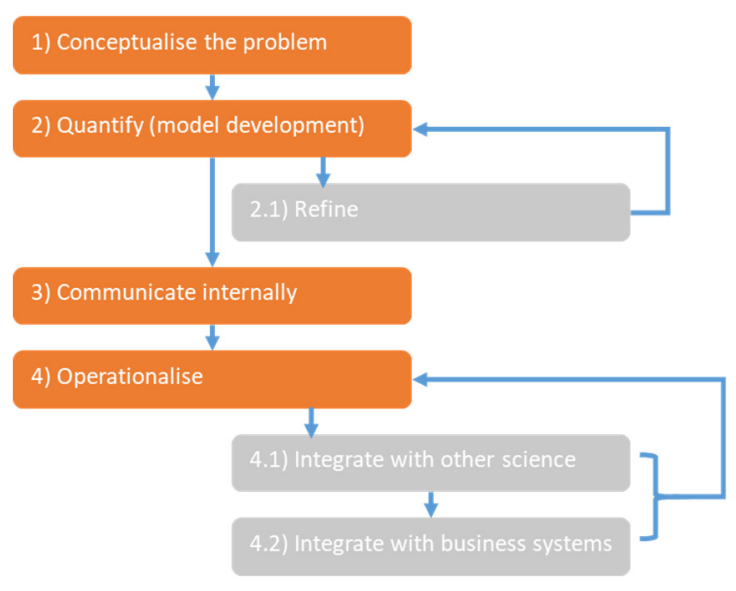

Figure 1. Workflow of modelling to support natural resource management 
Marsh et al., Visualising spatio-temporal modelling results: Getting modelling results into the hands of decision makers

Through the subsequent sections of this paper we describe the phases of the workflow. We illustrate the application of a progressive disclosure approach (using web based data visualisations) to delivering audienceappropriate content for each of the phases.

Table 1. Key workflow phases, purpose and audience

\begin{tabular}{|l|l|l|}
\hline Phase & Purpose & Audience \\
\hline 1) Conceptualise & $\begin{array}{l}\text { A shared appreciation of key processes and prioritisation of } \\
\text { where a numerical modelling effort is required. } \\
\text { Agreement on key variables and scenario alternatives }\end{array}$ & $\begin{array}{l}\text { Stakeholders in management } \\
\text { outcomes, Policy officers, Scientists, } \\
\text { Modellers }\end{array}$ \\
\hline 2) Quantify & Develop a fit-for-purpose numerical modelling solution. & Modellers \\
\hline 3) Communicate internally & Communicate the numerical model output to decision makers. & Modellers, Policy officers \\
\hline 4) Operationalise & $\begin{array}{l}\text { Communicate the planning decision process within the context } \\
\text { of complementary / competing policy and scientific issues. }\end{array}$ & $\begin{array}{l}\text { Stakeholders in management } \\
\text { outcomes, Policy officers, Modellers }\end{array}$ \\
\hline
\end{tabular}

\section{PHASE 1: CONCEPTUALISE THE PROBLEM}

The first phase of the workflow is to gain a shared understanding of the problem. This process is important to engage policy makers and stakeholders in a broader understanding of the complex interactions involved in natural resource management. It also plays a critical role in communicating the pathways and process understanding from the researcher to the modeller who must quantitatively represent these interactions. Conceptualising the problem ultimately defines the scope of the modelling process.

\subsection{Current practice}

The current approach for this phase of the process is to use conceptual modelling, whereby all relevant elements of the problem are represented pictorially. This approach successfully communicates all the important elements in a process but fails to adequately represent complex and multilevel interactions.

\subsection{Example: Interactive Conceptual modelling}

This example has been developed from a conceptual modelling exercise conducted by Alluvium Consulting to support Melbourne Water. The project required the development of conceptual models to allow Melbourne Water's stakeholders to more actively engage in their Healthy Waterways Strategy development process. A single picture based conceptual model is inadequate because it does not illustrate the relative strength, direction and underlying scientific understanding of different parts of the system. A further requirement was to allow the creation of conceptual models which can be applied to different landscape settings (e.g. upland vs estuary). Additionally the conceptual models had to be able to focus on individual environmental values.

Alluvium created a taxonomic system for the creation of the models. The taxonomic system is based on nodes and links. Nodes represent measurable end points or intermediate stages (e.g. fish abundance, hydrologic alteration). Links represent the process which describes the interaction between the nodes. There are a collection of different node types to allow grouping of end points (e.g. all environmental values, all hydrological changes). The grouping of nodes allows the model to be collapsed or expanded, allowing progressive disclosure of the underlying processes related each group of related nodes. Each link has properties of strength, direction and scientific rigor. Both nodes and links have a collection of contextual information which describes the source of the information and provides a plain English description. This structured collection of node and link properties lends itself well to creating a dynamic progressive disclosure visualisation which allows the users to explore the conceptual relationships.

The complex conceptual model (Figure 2) shows all nodes and links. The link colour represents the influence (red=negative, green=positive). The thickness of the link represents the strength of the association and the stroke type (solid, dashed) represents the underlying scientific confidence in the relationship. Clicking on any node, highlights that node and the direct connections to that node and presents a long form description of the node. 
Marsh et al., Visualising spatio-temporal modelling results: Getting modelling results into the hands of decision makers

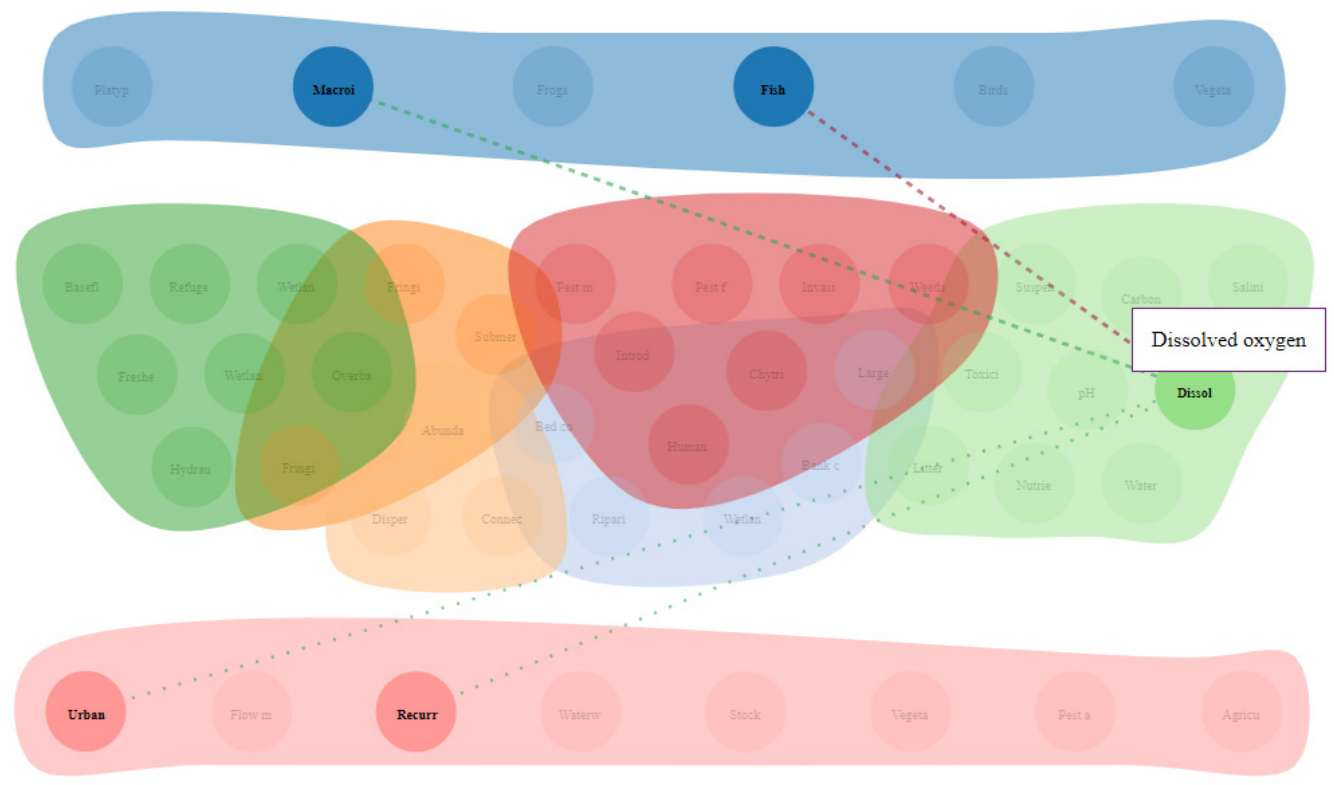

Figure 2. Interactive Conceptual model (showing all nodes and highlighted interaction path for dissolved oxygen)

\section{PHASE 2: QUANTIFY (BUILD AND REFINE THE PREDICTIVE MODEL)}

The second phase of the modelling to support natural resource management workflow is to create the numerical representation of the system. This is a huge component of the process but is largely focused on a single audience (modellers). These modellers are less interested in creating communication tools, but are focused on tools that allow the modelling team to rapidly share their model calibration results and compare and review scenarios and parameter sets.

\subsection{Current practice}

Model development and calibration is usually conducted by a single modeller. The process of rapid and iterative model calibration and testing does not lend itself well to constant team based review and interaction. This is largely due to the overheads of extracting and summarising key model results to share and discuss. This limitation greatly constrains the ability of teams to scale for large problems and to work from remote locations.

\subsection{Example: Office of Great Barrier Reef catchment modelling support}

The Queensland Government employs a team of $20+$ catchment modellers and technical support staff to quantify the impact of land management change on the Great Barrier Reef (GBR). These modellers use the eWater SOURCE catchment modelling platform (Carr and Podger 2012) to produce catchment models in each of six GBR regions, which collectively covers the $450,000 \mathrm{~km}^{2}$ which discharges to the GBR. The team is spread across several regional offices.

Truii.com has been working with the GBR catchment modelling team to look at ways to allow easier and faster interaction between remotely located modellers. Through this project Truii.com has developed a collection of functionality to allow automation of modelling post-processing activities and the automated generation of model calibration reports and output data visualisations (Figure 3). The consistent 'templating' approach to producing internal model performance reports ensures a consistent and repeatable internal reporting process.

The progressive disclosure approach for this case study is in the form of a nested series of visualisations which represent a hierarchy of data aggregation. At the highest level the spatial summary shows long term average values, followed by annual totals and further investigation by the user can reveal monthly values. 
Marsh et al., Visualising spatio-temporal modelling results: Getting modelling results into the hands of decision makers

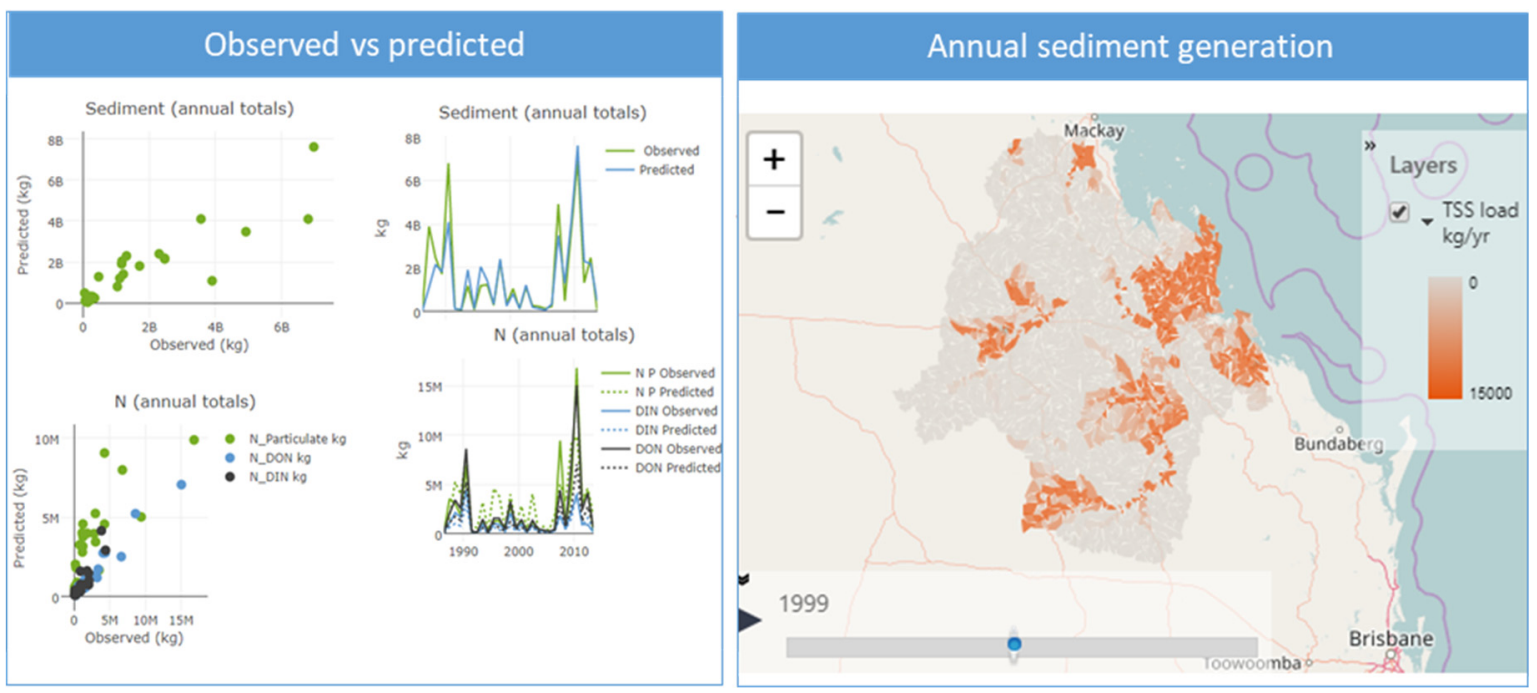

Figure 3. Example template web page of observed vs predicted model results and spatio-temporal data visualisation showing model output through time and space

\section{PHASE 3: COMMUNICATE INTERNALLY}

As the modelling process matures, it is time to re-engage with the client who commissioned the model.

\subsection{Current practice}

The current practice is usually to produce a calibration report and a summary report showing plots of 'typical' outputs. This is usually followed up with a series of workshops and presentations as it becomes clear that the information presented in the report isn't well directed to the business needs of the policy officer. This process of interaction can be unsatisfying in that the policy officer does not know what data to request because they don't know what is available. It is also unsatisfying for the modeller due to the repeated requests for data in different forms (maps, tables, charts) and levels of aggregation which requires a lot of manual handling.

\subsection{Example: South East Queensland catchment and estuarine modelling}

BMT WBM developed an extensive series of catchment (SOURCE) and linked estuarine (TUFLOW FV) models for the south east Queensland region for Healthy Land and Water (HLW). HLW is a consortium of 35 partner organisations including all the regions local authorities, state government, water supply providers, research organisations and several industry organisations. Collectively this group have varied interests and motivations in commissioning the modelling activity. The modelling effort is an ongoing concern with the development and implementation of scenarios to consider alternative land management strategies for south east Queensland.

This case study is focused on a single process; 'the development of land management scenarios' to apply to the models. A workshop was conducted with all the partner organisations to help collectively develop these future modelling scenarios. For this workshop the challenge was to deliver the results of models from multiple regions which represent catchment processes, estuarine process and receiving water impacts (Moreton Bay). Each member of the audience had a specific geographic focus or process focus.

To facilitate this scenario development process Truii.com worked with BMT WBM to develop a large collection of web pages (34) each with a collection of data visualisations (165 in total). The progressive disclosure approach was to group visualisation firstly by key process and then by region with a simple navigation page to access the underlying visualisations. For the workshop, individuals and groups investigated their specific region and process of interest in parallel by exploring the web-based data visualisations. The result was an efficient collation and prioritisation of scenarios to consider. The supporting web pages were useful after the workshop event for individuals from each organisation to be able to further brief their individual organisations on the process. 
Marsh et al., Visualising spatio-temporal modelling results: Getting modelling results into the hands of decision makers
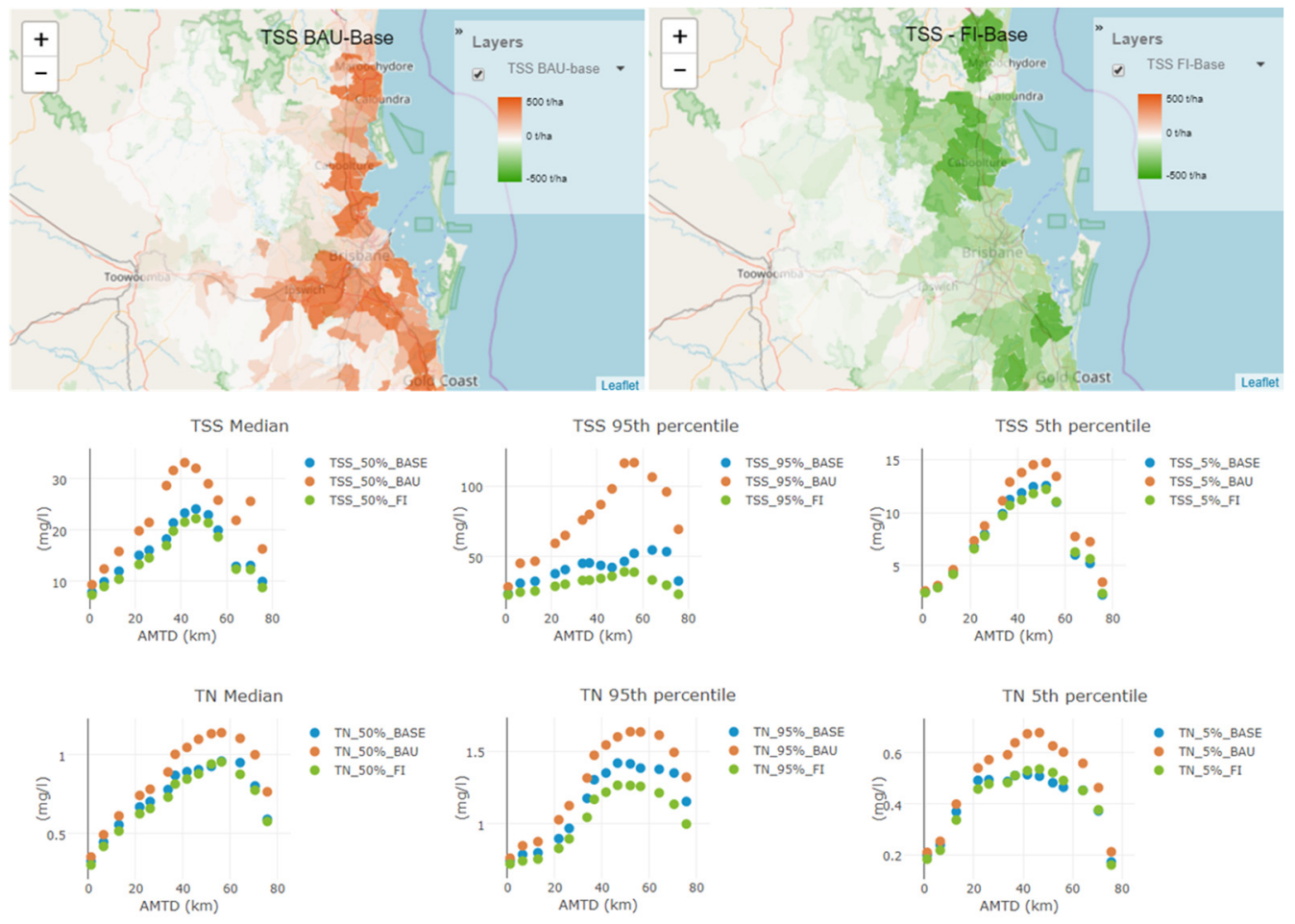

Figure 4. Catchment and estuarine model results for scenario workshop (Example spatial view -top and longitudinal view -bottom)

\section{PHASE 4: OPERATIONALISE}

The previous phases contributed to the development of modelling output that is fit for purpose for the natural resource manager. However, the planning and operational process rarely involves output from a single modelling process. There are outputs from other models, observational data, social and business unit processes which need to be wound around the model output so that it can be implemented in a planning or operational framework.

\subsection{Current practice}

The current practice for implementing modelling results into active natural resource management processes largely relies on skills of the natural resource manager to integrate all these information streams into an implementable solution. This usually occurs within the framework of advisory panels to produce a rather opaque 'best available science' approach. Whilst this efficient and pragmatic approach is to be applauded. In the increasingly scrutinised arena of where public resources are spent, a more quantitative, defensible and repeatable process is required.

\subsection{Example: Seqwater Catchment Investment Decision Support System (CIDSS)}

Seqwater supplies drinking water to 3.1M people in south East Queensland. Their source catchments are both complex multiuse catchments $(1.2 \mathrm{M} \mathrm{Ha})$, operate 22 water treatment plants and take their catchment stewardship very seriously. Seqwater have invested extensively in catchment modelling and data collection. They invest directly and in partnership with others in a large range of intervention strategies to improve catchment health. The problem addressed in this case study is "how can Seqwater better integrate the catchment modelling outputs and data collection programs to prioritise the type and location of on-ground investments. Truii.com has partnered with Seqwater to develop the Catchment Investment Decision Support System (CIDSS).

The CIDSS integrates SOURCE model outputs with point source observation data, microbial risk survey data and spatial attributes of the region. The CIDSS produces a spatially optimized (simulate annealing approach) scenario of on-ground interventions which maximize water quality improvements within a specified budget.

The CIDSS has a series of interfaces tailored to different audiences (whole organization, management, policy officers, planners, operations staff). 
Marsh et al., Visualising spatio-temporal modelling results: Getting modelling results into the hands of decision makers

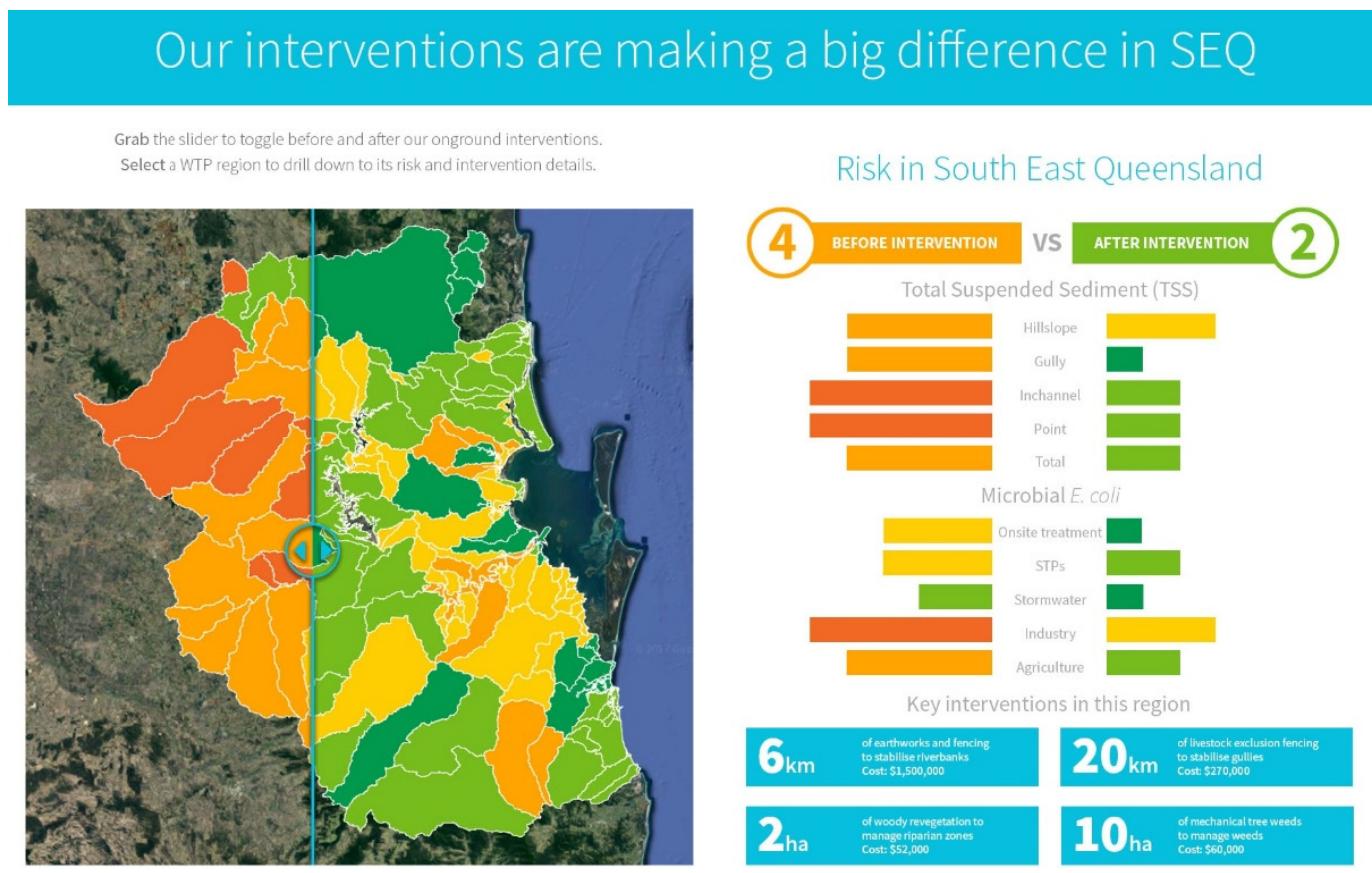

Figure 5. Catchment Investment Decision Support System main interface.

\section{CONCLUSIONS}

Natural resource management is a data driven industry with a high reliance on modelling. The methods of communicating model results has largely been based on producing document-based reports. We have presented a series of case studies where progressive natural resource management organisations are apply modern communication technologies to get a greater return from their modelling investment through better delivery of the modelling results. Web enabled data visualization and cloud delivery allows opportunities to deliver high data volume, more timely and through progressive disclosure.

\section{ACKNOWLEDGEMENTS}

Many thanks to those who have helped to create the above examples. Truii.com (Tory Grice, Sylvain Arene, Viet Phung, Bonny Marsh); Melbourne Water (Rob Molloy); Alluvium (Dom Blackham, Ross Hardy, Mark Stacey, Amanda Shipp, Barry Hart); Healthy Waterways (Paul Maxwell, James Udy); Seqwater (Andrew Smolders, Chris Thompson); Office the Great Barrier Reef modelling teams (Robin Ellis, Shawn Darr).

\section{REFERENCES}

Brugnach, M, and Pahl-Wostl, C (2008). A broadened view on the role for models in natural resource management: Implications for model development. pp 187-203 In. Pahl-Wostl, C., Kabat, P., and Möltgen, J. Adaptive and Integrated Water Management: Coping with Complexity and Uncertainty. Springer Berlin Heidelberg.

Carr, R., and Podger, G. (2012). eWater source - Australia's next generation IWRM modelling platform. In: Hydrology and Water Resources Symposium 2012. Barton, ACT: Engineers Australia, 2012: 742-749.

Cillers, P., Biggs, H., Blignaut, S., Choles, A, Hofmeyr, J., Jewitt, G, and Roux, D. (2013). Complexity, Modeling, and Natural Resource Management. Ecology and Society; Vol 18:3

Lidwell, W, Holden, K, and Butler, J. (2003). Universal principles of design: second edition. Rockport Publishers, Beverly Massachusetts. pp272.

McAfee, A, and Brynjolfsson E. (2012). Big Data: the management revolution, Harvard Business review, October 2012 issues (https://hbr.org/2012/10/big-data-the-management-revolution/ar)

Pandey, A., Manivannan, A., Nov, O., Satterthwaite, M., and Bertini, E. (2014). The Persuasive Power of Data Visualization. New York University Public Law and Legal Theory Working Papers. Paper 474. 